\title{
Prognostic value of dobutamine stress echocardiography in patients with previous coronary revascularisation
}

\author{
M Bountioukos, A Elhendy, R T van Domburg, A F L Schinkel, J J Bax, B J Krenning, E Biagini, \\ V Rizzello, M L Simoons, D Poldermans
}

Heart 2004;90:1031-1035. doi: 10.1136/hrt.2003.029025

See end of article for authors' affiliations

Correspondence to:

Correspondence to:
Dr Don Poldermans Thoraxcentre Room Ba 300, Department of Cardiology, Erasmus Medical Centre, $\mathrm{Dr}$ Molewaterplein 40, 3015 GD Rotterdam, the Netherlands; d.poldermans@ erasmusmc.nl

Accepted 7 February 2004

\begin{abstract}
Objective: To assess the prognostic value of dobutamine stress echocardiography (DSE) in patients with previous myocardial revascularisation.

Design: Prospective study.

Setting: Tertiary referral centre in Rotterdam, the Netherlands.

Patients: 332 consecutive patients with previous percutaneous or surgical coronary revascularisation underwent DSE. Follow up was successful for 331 (99.7\%) patients. Thirty eight patients who underwent early revascularisation ( $\leqslant 3$ months) after the test were excluded from analysis.

Main outcome measures: Cox proportional hazards regression models were used to identify independent predictors of the composite of cardiac events (cardiac death, non-fatal myocardial infarction, and late revascularisation).

Results: During a mean (SD) of 24 (20) months, 37 (13\%) patients died and $89(30 \%)$ had at least one cardiac event $(21(7 \%)$ cardiac deaths, $11(4 \%)$ non-fatal myocardial infarctions, and 68 (23\%) late revascularisations). In multivariate analysis of clinical data, independent predictors of late cardiac events were hypertension (hazard ratio (HR) 1.7, 95\% confidence interval (Cl) 1.1 to 2.6) and congestive heart failure (HR 2.1, 95\% Cl 1.3 to 3.2). Reversible wall motion abnormalities (ischaemia) on DSE were incrementally predictive of cardiac events (HR 2.1, 95\% Cl 1.3 to 3.2 ).

Conclusions: Myocardial ischaemia during DSE is independently predictive of cardiac events among patients with previous myocardial revascularisation, after controlling for clinical data.
\end{abstract}

\section{METHODS}

\section{Patient population}

The study population consisted of 332 consecutive patients with previous coronary revascularisation. Patients were unable to perform an exercise test because of orthopaedic limitations, peripheral arterial or neurological diseases, respiratory insufficiency, or deconditioning and they underwent DSE in our centre. If patients underwent DSE more than once after revascularisation, only the results of the first test were included in the study. All patients were enrolled in an electronic registry that accumulated in the course of daily clinical care. Informed consent was given before testing. The hospital ethics committee approved the protocol.

\section{Clinical data}

Before the dobutamine stress test, a structured interview and clinical history, including assessment of cardiac risk factors, were obtained. Congestive heart failure was assessed as any current or previous signs or symptoms of breathlessness, abnormal fluid retention, or both. Hypertension was defined as a blood pressure of $\geqslant 140 / 90 \mathrm{~mm} \mathrm{Hg}$ or treatment with antihypertensive medication. Diabetes mellitus was defined as a fasting glucose concentration of $\geqslant 140 \mathrm{mg} / \mathrm{dl}$ ( $\geqslant 7.8 \mathrm{mmol} / \mathrm{l}$ ) or the need for insulin or oral hypoglycaemic agents. Hypercholesterolaemia was defined as total cholesterol concentration of $\geqslant 200 \mathrm{mg} / \mathrm{dl}(\geqslant 5.2 \mathrm{mmol} / \mathrm{l})$ or treatment with lipid lowering medication.

\section{Dobutamine stress protocol}

Dobutamine stress testing was performed according to a standard protocol as previously reported. ${ }^{6}$ Dobutamine was administered intravenously, starting at a dose of $10 \mu \mathrm{g} / \mathrm{kg} /$ min for three minutes $(5 \mu \mathrm{g} / \mathrm{kg} / \mathrm{min}$ for patients with resting left ventricular dysfunction). Incremental dobutamine doses of $10 \mu \mathrm{g} / \mathrm{kg} / \mathrm{min}$ were given at three minute intervals up to a maximum dose of $40 \mu \mathrm{g} / \mathrm{kg} / \mathrm{min}$. If the test end point was not reached at a dobutamine dose of $40 \mu \mathrm{g} / \mathrm{kg} / \mathrm{min}$, atropine (up to $2 \mathrm{mg}$ ) was given intravenously. Blood pressure, heart rate, and ECG were constantly monitored. Test end points were achievement of the target heart rate $(85 \%$ of maximum age and sex predicted heart rate), horizontal or downsloping ST segment depression $>2 \mathrm{~mm}$ at an interval of $80 \mathrm{~ms}$ after the $\mathrm{J}$ point compared with baseline, severe angina, a 
systolic blood pressure fall $>40 \mathrm{~mm} \mathrm{Hg}$, blood pressure $>240 / 120 \mathrm{~mm} \mathrm{Hg}$, or significant cardiac arrhythmia. An intravenous $\beta$ blocker was available to reverse the adverse effects of dobutamine or atropine.

\section{Stress echocardiography}

Two dimensional echocardiographic images were acquired at rest, during dobutamine stress, and during recovery. The echocardiograms were recorded in a quad screen format. Two experienced observers scored the echocardiograms according to a standard 16 segment model as recommended by the American Society of Echocardiography. ${ }^{7}$ Regional wall motion and systolic wall thickening were scored on a five point scale ( 1 , normal; 2, mild hypokinesia; 3, severe hypokinesia; 4, akinesia; 5, dyskinesia). Ischaemia was defined as new or worsened wall motion abnormalities during stress, indicated by an increase of wall motion score $\geqslant 1 \operatorname{grade}(\mathrm{s})$ in $\geqslant 1$ segment(s). Ischaemia was not considered to be present when akinetic segments at rest became dyskinetic during stress. For each patient, a wall motion score index was calculated at rest and at peak dobutamine stress by dividing the sum of segments scored by the total number of interpreted segments.

\section{Follow up}

Follow up data were obtained in 2003. The mean (SD) follow up period was 24 (20) months. The current status was determined by contacting the patient's general practitioner and by reviewing hospital records. The date of the last review or consultation was used to calculate the follow up time. An outcome event was the composite of cardiac death, non-fatal myocardial infarction, and late $(>3$ months) coronary revascularisation. Cardiac death was defined as death caused by acute myocardial infarction, significant cardiac arrhythmias, or refractory congestive heart failure. Sudden death occurring without another explanation was regarded as cardiac death. Non-fatal myocardial infarction was defined according to the guidelines of the joint European Society of Cardiology/American College of Cardiology committee. ${ }^{8}$

\section{Statistical analysis}

Data were expressed as mean (SD) or number (\%) and compared by Student's $t$ test or the $\chi^{2}$ test. Univariate and multivariate Cox proportional hazards regression models were used to identify independent predictors of late cardiac events. The clinical variables that were entered into the model were sex, age, history of previous myocardial infarction, current smoking, hypertension, hypercholesterolaemia, heart failure, angina pectoris, and the use of $\beta$ blockers, diuretics, digoxin, calcium antagonists, and angiotensin converting enzyme antagonists. The echocardiographic variables were fixed wall motion abnormalities, reversible wall motion abnormalities, or both, achievement of target heart rate, and typical angina or ST depression during the stress test. Variables were selected in a stepwise forward selection manner with entry and retention set at a significance level of 0.05 . The incremental value of DSE over the clinical variables in the prediction of events was assessed by adding various echocardiographic data to clinical and stress test parameters. The risk of a variable was expressed as a hazard ratio (HR) with a corresponding 95\% confidence interval (CI). The probability of cardiac event-free survival was calculated by the Kaplan-Meier method and the resulting curves were compared by the log rank test.

\section{RESULTS}

\section{Clinical and stress test results}

Table 1 presents the clinical characteristics of patients. During DSE there was a significant increase in heart rate (from 74 (14) to 132 (15) beats/min, p < 0.001), whereas systolic blood pressure did not change significantly (131 (24) $v 129$ (24) $\mathrm{mm} \mathrm{Hg}$ ). The mean (SD) maximum dobutamine dose was $37(8) \mu \mathrm{g} / \mathrm{kg} / \mathrm{min}$. Atropine was added for 153 $(52 \%)$ patients. Target heart rate was achieved by $252(86 \%)$ patients. Side effects during DSE were short runs of ventricular tachycardia $(<10$ complexes $)$ in $10 \quad(3 \%)$ patients, ventricular tachycardia of $\geqslant 10$ complexes in three $(1 \%)$, transient atrial fibrillation in three $(1 \%)$, and severe hypotension (symptomatic or decrease in systolic blood pressure of $>40 \mathrm{~mm} \mathrm{Hg}$ ) in four $(1 \%)$. No patient experienced myocardial infarction or life threatening rhythm disorders.

\section{Echocardiographic data}

The test was abnormal (fixed or reversible wall motion abnormalities or both) in $241(82 \%)$ patients. Ischaemia (new or worsening wall motion abnormalities) was detected in $96(33 \%)$ patients. Of these patients, 75 had resting wall motion abnormalities as well.

\section{Follow up data}

Follow up was successful for 331 (99.7\%) patients. The decision for late revascularisation was based on the recurrence of symptomatic coronary artery disease or on the occurrence of an acute coronary event (unstable angina, acute myocardial infarction) at least three months apart from the first post-revascularisation DSE.

Thirty eight patients who underwent coronary revascularisation within three months of DSE were excluded from analysis because for these patients the decision to revascularise might have been influenced by test results. Among the remaining 293 patients, 37 (13\%) died of any cause and 89 (30\%) patients had at least one cardiac event; 21 (7\%) patients had a cardiac death, 11 (4\%) had a non-fatal myocardial infarction, and $68(23 \%)$ had a late revascularisation.

\section{Predictors of cardiac events}

Univariate predictors of the composite of cardiac events were congestive heart failure (HR 2, 95\% CI 1 to 3.8), wall motion score index at rest (HR 2.7, 95\% CI 1.4 to 5), wall motion score index at peak (HR 2, 95\% CI 1.2 to 3.3), reversible wall motion defects (ischaemia) on DSE (HR 2.9, 95\% CI 1.7 to 4.8 ), ST segment depression (HR 2.8, 95\% CI 1.2 to 6.3 ), and angina pectoris (HR 2, 95\% CI 1.2 to 3.5 ) during the test.

Table 1 Baseline clinical characteristics of 293 patients

\begin{tabular}{ll}
\hline Characteristic & $\begin{array}{l}\text { Mean (SD) or } \\
\text { number (\%) }\end{array}$ \\
\hline Age (years) & $62(11)$ \\
Men & $217(74 \%)$ \\
Hypertension & $119(40 \%)$ \\
Diabetes mellitus & $97(33 \%)$ \\
Smoking & $97(33 \%)$ \\
Hypercholesterolaemia & $120(41 \%)$ \\
Congestive heart failure & $46(16 \%)$ \\
Myocardial infarction & $203(69 \%)$ \\
Coronary angioplasty & $174(59 \%)$ \\
Coronary artery bypass surgery & $171(58 \%)$ \\
1 vessel disease & $141(48 \%)$ \\
2 vessel disease & $94(32 \%)$ \\
3 vessel or left main disease & $58(20 \%)$ \\
Complete revascularisation & $202(69 \%)$ \\
Incomplete revascularisation & $91(31 \%)$ \\
Medication & $127(43 \%)$ \\
$\quad \beta$ Blockers & $128(44 \%)$ \\
ACE inhibitors & $121(41 \%)$ \\
Calcium antagonists & \\
\hline ACE, angiotensin converting enzyme. &
\end{tabular}




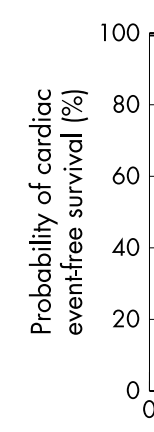

Number at risk

Non-ischaemic DSE 194

Ischaemic DSE
95
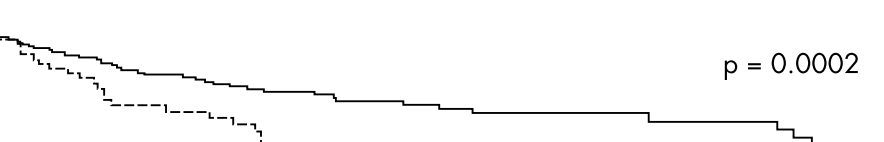

.
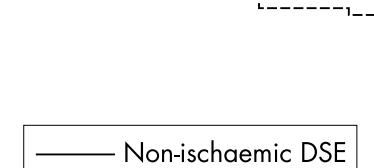

----- Ischaemic DSE

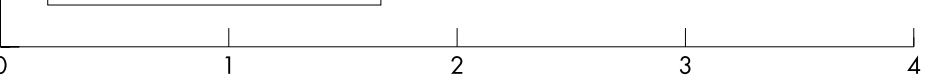

Years of follow up

Multivariate predictors were hypertension (HR 1.7, 95\% CI 1.1 to 2.6), congestive heart failure (HR 2.1, 95\% CI 1.3 to 3.2), ischaemia on DSE (HR 2.1, 95\% CI 1.3 to 3.2) (fig 1 ), and ST segment depression on DSE (HR 2, 95\% CI 1.1 to 3.8). Among patients with ischaemia on DSE, those who had had a coronary intervention $<2$ years before the test had a trend towards more cardiac events compared with patients who had undergone revascularisation $>2$ years before $(\mathrm{p}=0.09)$ (fig 2). The adverse outcome for patients with ischaemia was equal irrespective of the presence or absence of angina before the test (fig 3).

\section{Predictors of cardiac death}

Univariate predictors of cardiac death were diabetes mellitus (HR 3.6, 95\% CI 1.2 to 11.4) and congestive heart failure (HR $6.8,95 \%$ CI 2.8 to 16.3 ). The same predictors were found after multivariate analysis (HR 4.9, 95\% CI 1.4 to 17.3 for diabetes mellitus and HR 10.5, 95\% CI 3.8 to 29.3 for congestive heart failure).

\section{Predictors of hard events}

The only predictor of hard events (cardiac death plus myocardial infarction) was congestive heart failure (HR 4.7, 95\% CI 2.3 to 9.6 in univariate analysis and HR 8.1, 95\% CI 3.5 to 18.5 in multivariate analysis).

\section{DISCUSSION}

We assessed the independent value of DSE for prediction of cardiac events in 331 patients with previous revascularisation.

During a mean period of 24 (20) months, 89 patients had at least one late cardiac event. The presence of congestive heart failure predicted cardiac death or myocardial infarction, whereas diabetes mellitus was an independent predictor of cardiac death. Clinical predictors of cardiac events were hypertension and congestive heart failure. Ischaemia on DSE, indicated by either reversible wall motion abnormalities or ST segment depression, was associated with increased risk of cardiac events after controlling for clinical parameters. According to the classic ischaemic cascade, ST segment changes follow perfusion and wall motion abnormalities on imaging stress testing. However, Picano ${ }^{9}$ has proposed an alternative ischaemic cascade when endothelial dysfunction and impaired coronary flow reserve are present without significant epicardial coronary artery lesions. According to this model, ST segment changes come first, perfusion abnormalities come second, and echocardiographic changes are usually absent in the case of milder, "patchy" degrees of myocardial ischaemia. Hence, coronary endothelial dysfunction and impaired flow reserve are likely to have an adverse effect on prognosis after coronary revascularisation.

The risk associated with myocardial ischaemia was observed among patients who had their revascularisation performed within two years, as well as among patients who had been revascularised $>2$ years before DSE.

\section{Impact of symptoms on outcome}

Restenosis is the major limitation of coronary interventions. It occurs usually within several months after a successful

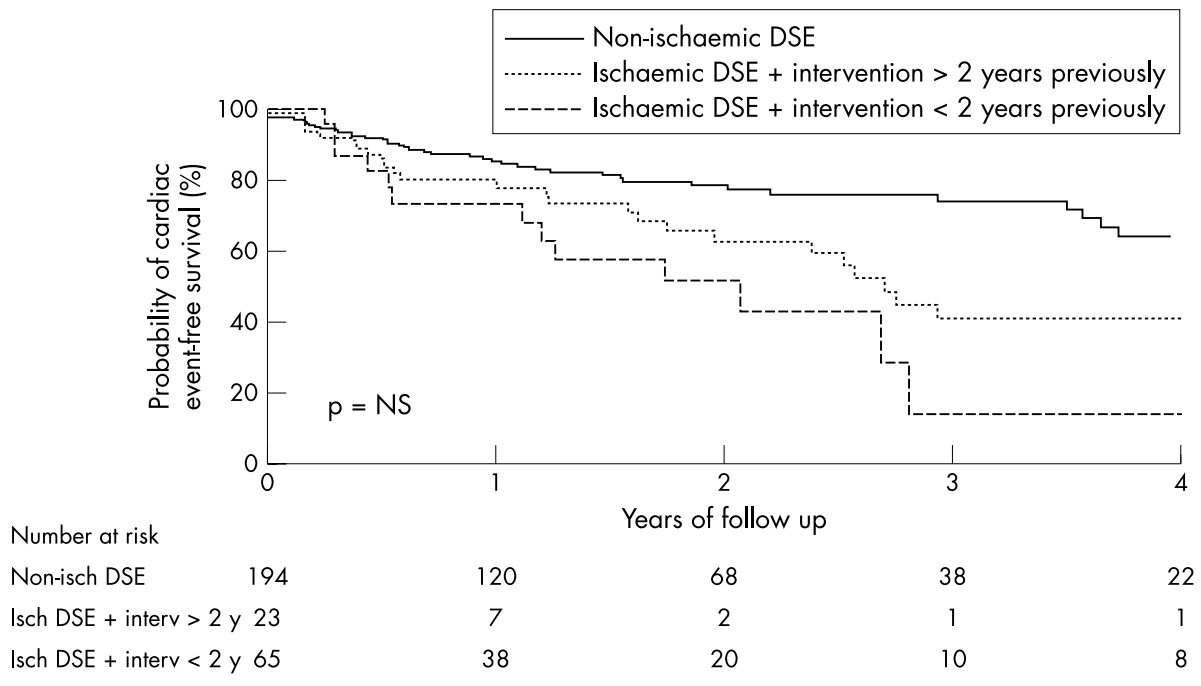

Figure 2 Kaplan-Meier curves for cardiac events (cardiac death, non-fatal myocardial infarction, late revascularisation) as a function of DSE results. Among patients with reversible wall motion defects (ischaemia) eventfree survival did not differ significantly between patients who were revascularised $>2$ years and patients who were revascularised $<2$ years before the test. NS, not significant.

Number at risk

Non-isch DSE

38
20
1 


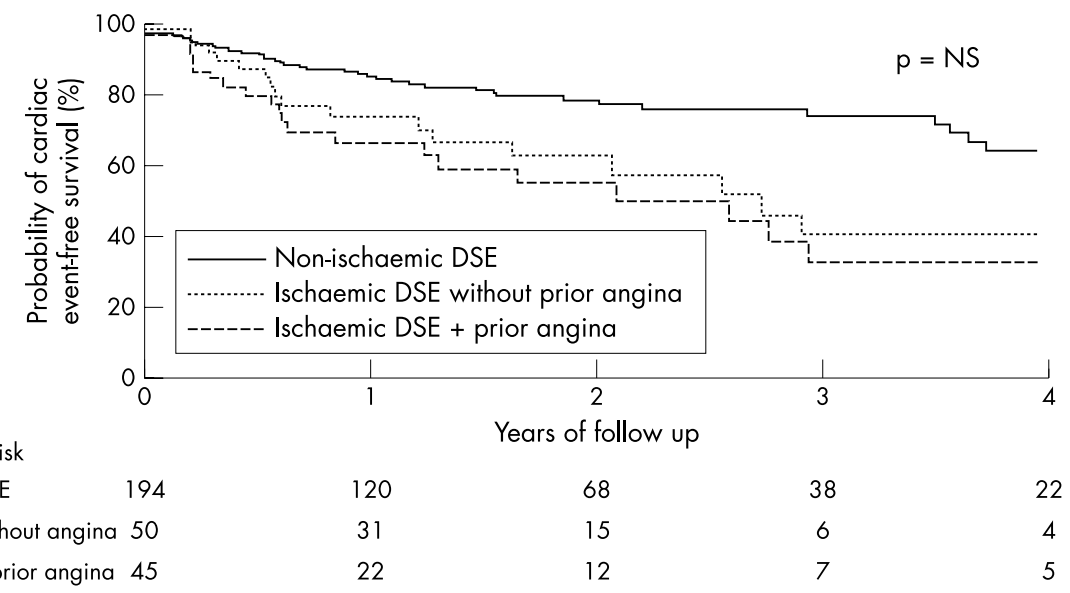

Figure 3 Kaplan-Meier curves for cardiac events (cardiac death, non-fatal myocardial infarction, late revascularisation) as a function of DSE results. Among patients with reversible wall motion defects (ischaemia) eventfree survival did not differ significantly between patients with and patients without a history of angina pectoris.

Number at risk

Non-isch DSE

Isch DSE without angina 50

Isch DSE + prior angina 45
3 years after coronary angioplasty ${ }^{24}$ and for patients early (5 (2) months) after coronary stenting. ${ }^{25}$ The present findings indicate that DSE can alternatively be used as a prognostic tool for these patients.

\section{Study limitations}

Readers of DSE results were not blinded to clinical information and data concerning previous revascularisations. Additionally, physicians were free to modify treatment according to DSE results. Thus, administration of medication that has been proved to reduce cardiac events and improve outcome, such as $\beta$ blockers and angiotensin converting enzyme inhibitors, might have influenced patient prognosis. Finally, our results were obtained in a single centre with a high volume and experience with DSE; hence, our results do not necessarily apply to other, less experienced centres.

\section{Conclusions}

DSE provides incremental data to the clinical information on the prognosis of patients after revascularisation. Evidence of myocardial ischaemia, based on reversible wall motion abnormalities during dobutamine infusion, is an independent predictor of cardiac events.

\section{Authors' affiliations}

M Bountioukos, A Elhendy, R T van Domburg, A F L Schinkel, B J Krenning, E Biagini, V Rizzello, M L Simoons, D Poldermans, Thoraxcentre, Department of Cardiology, Erasmus Medical Centre, Rotterdam, The Netherlands

J J Bax, Department of Cardiology, Leiden University Medical Centre, Leiden, The Netherlands

\section{REFERENCES}

1 Rubenson DS, Tucker CR, London E, et al. Two-dimensional echocardiographic analysis of segmental left ventricular wall motion before and after coronary artery bypass surgery. Circulation 1982;66:1025-33.

2 Pressman GS. Abnormal stress echocardiography results after coronary stenting. J Am Soc Echocardiogr 2001;14:948-50.

3 Hoffmann $R$, Lethen $H$, Falter $F$, et al. Dobutamine stress echocardiography after coronary artery bypass grafting: transthoracic vs biplane transoesophageal imaging. Eur Heart J 1996;17:222-9.

4 Kamaran M, Teague SM, Finkelhor RS, et al. Prognostic value of dobutamine stress echocardiography in patients referred because of suspected coronary artery disease. Am J Cardiol 1995;76:887-91.

5 Steinberg EH, Madmon L, Patel CP, et al. Long-term prognostic significance of dobutamine echocardiography in patients with suspected coronary artery disease: results of a 5-year follow-up study. J Am Coll Cardiol 1997;29:969-73

6 Poldermans D, Fioretti PM, Boersma E, et al. Long-term prognostic value of dobutamine-atropine stress echocardiography in 1737 patients with known or suspected coronary artery disease: a single-center experience. Circulation 1999;99:757-62

7 Armstrong WF, Pellikka PA, Ryan T, et al. Stress echocardiography: recommendations for performance and interpretation of stress within two years after surgical revascularisation. In other studies, ${ }^{201} \mathrm{Tl}$ was predictive of cardiac events for patients $1-3$ 
echocardiography. Stress echocardiography task force of the nomenclature and standards committee of the American Society of Echocardiography. J Am Soc Echocardiogr 1998; 11:97-104.

8 Alpert JS, Thygesen K, Antman E, et al. Myocardial infarction redefined: a consensus document of the joint European Society of Cardiology/American College of Cardiology committee for the redefinition of myocardial infarction. J Am Coll Cardiol 2000;36:959-69.

9 Picano E. The alternative "ischaemic" cascade in coronary microvascular disease. Cardiologia 1999;44:791-5.

10 Holmes DR Jr. State of the art in coronary intervention. Am J Cardiol 2003;91:50A-3A

11 Campeau L, Enjalbert M, Lesperance J, et al. Atherosclerosis and late closure of aortocoronary saphenous vein grafts: sequential angiographic studies at 2 weeks, 1 year, 5 to 7 years, and 10 to 12 years after surgery. Circulation 1983:68:III-7.

12 Gibbons RJ, Balady GJ, Timothy Bricker J, et al. ACC/AHA 2002 guideline update for exercise testing: summary article. A report of the American College of Cardiology/American Heart Association task force on practice guidelines (committee to update the 1997 exercise testing guidelines). J Am Coll Cardiol 2002;40:1531-40.

13 Laver MS, Lytle B, Pashkow F, et al. Prediction of death and myocardial infarction by screening with exercise-thallium testing after coronary-arterybypass grafting. Lancet 1998;351:615-22.

14 Sarda L, Fuchs L, Lebtahi R, et al. Prognostic value of 201Tl myocardia scintigraphy after coronary artery bypass grafting. Nucl Med Commun 2001;22:189-96.

15 Sozzi FB, Elhendy A, Roelandt JR, et al. Prognostic value of dobutamine stress echocardiography in patients with diabetes. Diabetes Care 2003;26:1074-8.

16 Williams MJ, Odabashian J, Laver MS, et al. Prognostic value of dobutamine echocardiography in patients with left ventricular dysfunction. J Am Coll Cardiol 1996;27:132-9.
17 Greco CA, Salustri A, Seccareccia F, et al. Prognostic value of dobutamine echocardiography early after uncomplicated acute myocardial infarction: a comparison with exercise electrocardiography. J Am Coll Cardiol 1997;29:261-7.

18 Sitges M, Pare C, Azqueta M, et al. Feasibility and prognostic value of dobutamine-atropine stress echocardiography early in unstable angina. Eur Heart J 2000;21:1063-71

19 Bholasingh R, Cornel JH, Kamp O, et al. Prognostic value of predischarge dobutamine stress echocardiography in chest pain patients with a negative cardiac troponin T. J Am Coll Cardiol 2003;41:596-602.

20 Poldermans D, Arnese M, Fioretti PM, et al. Improved cardiac risk stratification in major vascular surgery with dobutamine-atropine stress echocardiography. J Am Coll Cardiol 1995;26:648-53.

21 Arruda AM, McCully RB, Oh JK, et al. Prognostic value of exercise echocardiography in patients after coronary artery bypass surgery. Am J Cardiol 2001;87:1069-73.

22 Palmas W, Bingham S, Diamond GA, et al. Incremental prognostic value of exercise thallium-201 myocardial single-photon emission computed tomography late after coronary artery bypass surgery. J Am Coll Cardiol 1995:25:403-9.

23 Miller TD, Christian TF, Clements IP, et al. Prognostic value of exercise thallium-201 imaging in a community population. Am Heart $J$ 1998;135:663-70.

24 Ho KT, Miller TD, Holmes DR, et al. Long-term prognostic value of Duke treadmill score and exercise thallium-201 imaging performed one to three years after percutaneous transluminal coronary angioplasty. Am J Cardiol 1999;84:1323-7.

25 Cottin Y, Rezaizadeh K, Touzery C, et al. Long-term prognostic value of 201Tl single-photon emission computed tomographic myocardial perfusion imaging after coronary stenting. Am Heart J 2001;141:999-1006.

\section{IMAGES IN CARDIOLOGY}

\section{Aortic arch aneurysm}

A 68 year old man was admitted to hospital for an ischaemic stroke. His medical history revealed hypertension, diabetes mellitus, chronic atrial fibrillation, and a blunt trauma sustained during a motor vehicle accident. Routine chest $x$ ray revealed an enlarged superior mediastinal silhouette (left panel). Subsequently, magnetic resonance imaging (MRI) showed a thoracic saccular aneurysm of the aortic arch with involvement of the branches (right panel).

Transverse arch aneurysms are relatively uncommon, comprising only 11\% of all aortic aneurysms. Saccular thoracic aneurysms are frequently posttraumatic (high speed deceleration accidents); other causes include syphilis, autoimmune disorders, and atherosclerosis. The most frequently involved location is the relatively immobile isthmus just distal to the origin of the left subclavian artery, adjacent to the attachment of the ligamentum arteriosus.

Many thoracic aneurysms are visible on chest $x$ ray and are characterised by widening of the mediastinal silhouette, enlargement of the aortic knob or

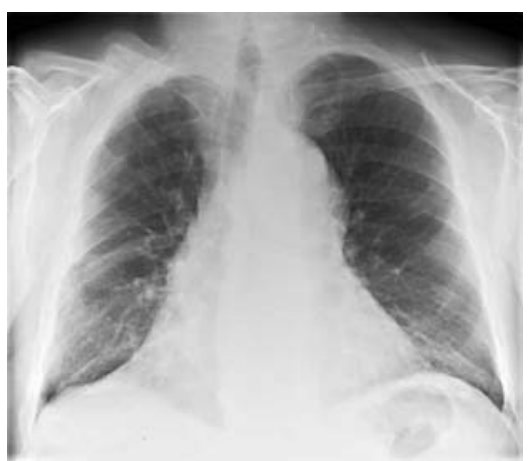

displacement of the trachea of the midline.

However, saccular aneurysms may not be evident on the chest $x$ ray.

MRI is useful in detecting aneurysms and especially magnetic resonance angiography may prove to be useful in defining the anatomy of aortic branch vessels. The aneurysm in this patient may be caused by atherosclerotic disease and/or his deceleration trauma.

K Bogaard

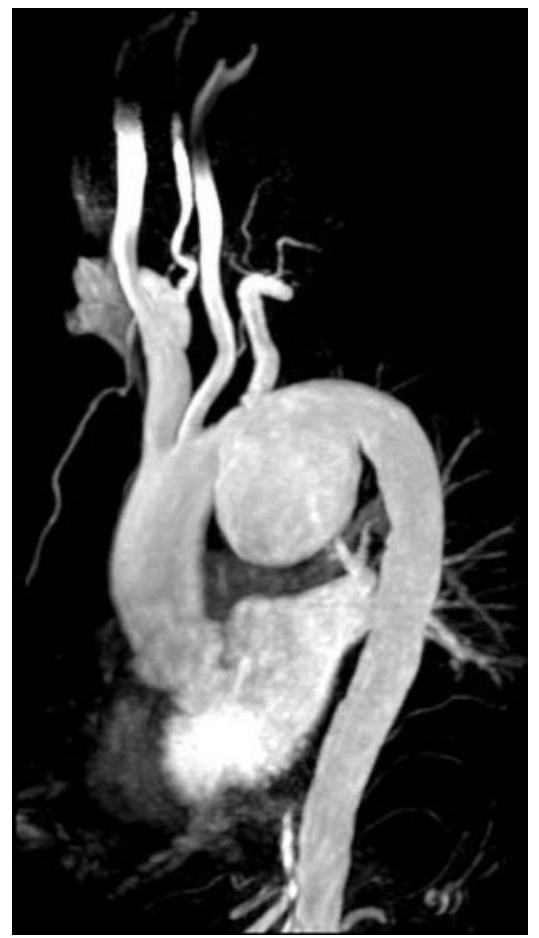

A J H A Scholte ajhascholte@freeler.nl 